\title{
OCT1 polymorphism is associated with response and survival time in anti-Parkinsonian drug users
}

\author{
Matthijs L. Becker • Loes E. Visser • \\ Ron H. N. van Schaik • Albert Hofman • \\ André G. Uitterlinden • Bruno H. Ch. Stricker
}

Received: 3 May 2010 / Accepted: 19 July 2010 /Published online: 1 August 2010

(C) The Author(s) 2010. This article is published with open access at Springerlink.com

\begin{abstract}
Substrates for the Organic Cation Transporter 1, encoded by the SLC22A1 gene, are metformin, amantadine, pramipexole, and, possibly, levodopa. Recently, we identified that the rs622342 A > C polymorphism is associated with the HbA1c lowering effect in metformin users. In the Rotterdam Study, we associated this polymorphism with higher prescribed doses of all anti-Parkinsonian drugs. Between the first and fifth prescriptions for levodopa, for
\end{abstract}

M. L. Becker · L. E. Visser · A. Hofman • A. G. Uitterlinden •

B. H. C. Stricker $(\bowtie)$

Department of Epidemiology, Erasmus MC,

P.O. Box 2040, 3000 CA Rotterdam, The Netherlands

e-mail: b.stricker@erasmusmc.nl

M. L. Becker · L. E. Visser

Hospital Pharmacy, Erasmus MC,

Rotterdam, The Netherlands

R. H. N. van Schaik

Department of Clinical Chemistry, Erasmus MC,

Rotterdam, The Netherlands

R. H. N. van Schaik

STAR Medical Diagnostic Center,

Rotterdam, The Netherlands

A. Hofman - A. G. Uitterlinden - B. H. C. Stricker The Netherlands Consortium on Healthy Aging, Leiden/Rotterdam, The Netherlands

A. G. Uitterlinden • B. H. C. Stricker

Department of Internal Medicine, Erasmus MC,

Rotterdam, The Netherlands

B. H. C. Stricker

Drug Safety Unit, Inspectorate for Health Care,

The Hague, The Netherlands each minor rs622342 $\mathrm{C}$ allele, the prescribed doses were 0.34 defined daily dose higher $(95 \%$ CI $0.064,0.62 ; p=$ $0.017)$. The mortality ratio after start of levodopa therapy was 1.47 times higher ( $95 \%$ CI 1.01, 2.13; $p=0.045$ ).

Keywords Organic Cation Transporter 1 Anti-Parkinson Agents $\cdot$ Pharmacogenetics $\cdot$ Pharmacoepidemiology

\section{Introduction}

Transporter proteins have a major role in the absorption, distribution, and elimination of a wide variety of compounds. Organic cation transporters (OCT) are involved in the carriage of both endogenous compounds, such as dopamine, and drugs, such as metformin and some antiParkinsonian drugs [1-3]. The three most important members of the OCT family are OCT1, OCT2, and OCT3, encoded by the SLC22A1, SLC22A2, and SLC22A3 genes, respectively. Pramipexole and amantadine are substrates for OCT1 and OCT2 [2, 4-6]. Levodopa is a substrate for OCT, although the subtype has not yet been identified [7, 8].

Recently, we identified that the polymorphism rs622342 $\mathrm{A}>\mathrm{C}$ in the SLC22A1 gene, coding for OCT1, was associated with the reduction in $\mathrm{HbAlc}$ levels in incident metformin users [9]. As this polymorphism might also be involved in the transportation efficacy of anti-Parkinsonian drugs, it may affect the response to these drugs. In this population-based cohort study, we studied whether prescribed doses of levodopa and co-prescribed antiParkinsonian drugs differed between rs622342 genotypes in incident levodopa users. We also studied the difference in survival time after start of levodopa therapy. 


\section{Methods}

Data for these analyses were obtained from the Rotterdam Study, a prospective population-based cohort study of 7,983 Caucasians aged 55 years and older, who were followed from 1990 onwards [10]. All participants of the Rotterdam Study gave written informed consent. Ethical approval was obtained from the Medical Ethical Committee of the Erasmus MC.

The study sample consisted of all subjects in the Rotterdam Study who had a first prescription for levodopa between July 1st 1991 and January 1st 2008. Participants who had prescriptions for levodopa between January 1st 1991 and July 1st 1991 were excluded to ensure that only incident levodopa users were included. We also excluded all participants who were prescribed less than three prescriptions for levodopa, because, most likely, levodopa treatment was used as a diagnostic tool. Participants were genotyped with the Illumina 550k SNP array [11].

Patients were followed from the first prescription of levodopa onwards, irrespective of whether they used other anti-Parkinsonian drugs at or before the date of the first levodopa prescription. We used two types of study outcome. First, for every prescription of levodopa, the change in prescribed daily dose of levodopa plus the dose of coprescribed other anti-Parkinsonian drugs (anticholinergic drugs, amantadine, bromocriptine, pergolide, ropinirole, pramipexole, selegiline, and entacapone) compared to the prescribed daily doses at the time of the first levodopa was calculated. To make the prescribed doses of different antiParkinsonian drugs comparable to each other, we divided the prescribed daily dose by the defined daily dose (DDD). The DDD is a standardized dosing measure representing the recommended daily dose for the main indication in an adult. The defined daily doses of controlled release levodopa prescriptions were multiplied by 0.7 to correct for differences in bioavailability. Associations between the number of rs622342 variant $\mathrm{C}$ alleles and changes in cumulative prescribed doses were analyzed using multivariate linear regression. We separately analyzed the associations between genotype and start of dopamine agonist therapy and time to discontinuation of levodopa therapy with Cox proportional hazard models. Second, we analyzed the difference between rs622342 genotypes in time from the first prescription for levodopa until death due to any cause with Cox proportional hazard models. Analyses were adjusted for age and sex.

\section{Results}

In the Rotterdam Study, 99 participants were identified who were incident levodopa users (Table 1). The minor allele frequency of rs622342 (C allele) was 0.36, and the genotype distribution was in Hardy-Weinberg equilibrium $(p=0.45)$. No statistically significant differences were found in time until the first prescription for levodopa in the whole Rotterdam Study, the prescribed doses at start of levodopa therapy, or use of other anti-Parkinsonian drugs at start of levodopa therapy. The average time that drugs were dispensed for per levodopa prescription was 52.5 days, and the rs622342 genotype was not associated with this duration.

The average prescribed dose of levodopa and coprescribed drugs increased after start of levodopa therapy. The increase was higher with the number of variant $\mathrm{C}$ alleles (Fig. 1). With each minor $\mathrm{C}$ allele, the change in prescribed daily dose of all anti-Parkinsonian drugs between the first and fifth prescriptions for levodopa was 0.34 DDD higher (95\% CI 0.064, 0.62). The prescribed daily dose of levodopa was 0.01 DDD higher $(95 \%$ CI $-0.035,0.062)$

Table 1 Baseline characteristics

\begin{tabular}{|c|c|c|c|c|}
\hline \multicolumn{2}{|l|}{ rs622342 } & AA & $\mathrm{AC}$ & $\mathrm{CC}$ \\
\hline \multicolumn{2}{|l|}{$N$} & 39 & 49 & 11 \\
\hline \multicolumn{2}{|l|}{ Gender, male $(\%)$} & $17(43 \%)$ & $22(45 \%)$ & $3(27 \%)$ \\
\hline \multicolumn{2}{|l|}{ Age, year (SD) } & $77.7(7.1)$ & $78.0(7.4)$ & $78.8(7.5)$ \\
\hline \multirow[t]{2}{*}{ Follow-up, year (SD) } & In Rotterdam Study & $11.8(4.5)$ & $10.0(4.1)$ & $9.4(5.0)$ \\
\hline & After start levodopa & $5.0(3.8)$ & $4.6(3.6)$ & $3.5(2.6)$ \\
\hline \multicolumn{2}{|l|}{ Body-mass index, $\mathrm{kg} / \mathrm{m}^{2}(\mathrm{SD})^{\mathrm{a}}$} & $28.2(6.3)$ & $26.2(3.9)$ & $27.6(4.8)$ \\
\hline \multicolumn{2}{|l|}{ Creatinine level, $\mu \mathrm{mol} / 1$ (SD) ${ }^{\text {a }}$} & $85.9(16.5)$ & $79.9(14.2)$ & $79.8(9.7)$ \\
\hline \multirow[t]{4}{*}{ Prior use before start levodopa therapy } & Anticholinergic drugs & $5(13 \%)$ & $7(14 \%)$ & $1(9 \%)$ \\
\hline & Dopamine agonists & $3(8 \%)$ & $4(8 \%)$ & $1(9 \%)$ \\
\hline & Amantadine & $12(31 \%)$ & $11(22 \%)$ & $5(45 \%)$ \\
\hline & Selegiline & $13(33 \%)$ & $15(31 \%)$ & $4(36 \%)$ \\
\hline \multicolumn{2}{|c|}{ Average prescribed dose of non-levodopa anti-Parkinsonian drugs at start levodopa therapy } & $0.84 \mathrm{DDD}$ & $0.80 \mathrm{DDD}$ & $0.68 \mathrm{DDD}$ \\
\hline
\end{tabular}

${ }^{a}$ At the time of entrance in the Rotterdam Study 


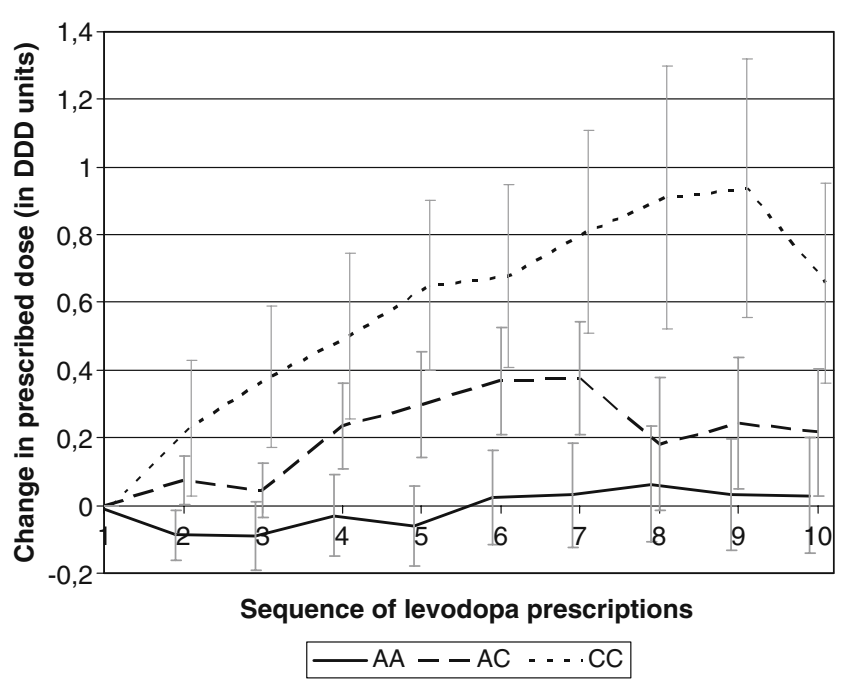

Fig. 1 Rs622342 genotype and change in prescribed doses of antiParkinsonian drugs (bars represent standard error)

and the prescribed daily dose of other anti-Parkinsonian drugs was 0.33 DDD higher $(95 \%$ CI $0.049,0.61)$. The change in prescribed daily dose was 0.038 DDD $(95 \%$ CI $-0.007,0.083)$ for the anticholinergic drugs, 0.11 DDD (95\% CI $0.01,0.20)$ for amantadine and 0.19 DDD $(95 \%$ CI $-0.07,0.45)$ for selegiline. Only six patients were prescribed dopamine agonists at the fifth prescription and in none of these patients the prescribed daily dose had changed between the first and fifth levodopa prescription. Therefore, changes in dopamine agonist doses did not contribute to the change in total prescribed daily dose of anti-Parkinson drugs. No significant associations were found between the rs622342 genotype and start of dopamine agonist therapy (HR 0.99 95\% CI 0.45, 2.19) or discontinuation of levodopa therapy (HR 1.13 95\% CI $0.72,1.76$ ).

The average survival time after start of levodopa therapy differed between rs622342 genotypes. The average survival time was 6.9 years for patients with the AA genotype, 5.2 years for patients with the AC genotype, and 4.4 years for patients with the $\mathrm{CC}$ genotype. The mortality ratio was significantly raised with the number of minor $\mathrm{C}$ alleles (HR 1.47 ; $95 \%$ CI 1.01, 2.13).

\section{Discussion}

In this population-based cohort study, the minor $\mathrm{C}$ allele at rs622342 in the SLC22A1 gene, encoding the transporter OCT1, was associated with higher prescribed doses of antiParkinsonian drugs and a shorter survival after start of levodopa therapy. This polymorphism was previously associated with metformin response and the rs622342 minor $\mathrm{C}$ allele is most likely associated with a less functioning OCT1 transporter. We could not exactly identify which drug or drugs contributed to the difference in prescribed daily dose of anti-Parkinsonian drugs. The only individual drug for which the prescribed daily doses were significantly associated with the rs622342 genotype was amantadine. We cannot exclude that the rs 622342 variant $C$ allele is associated with a lower response to levodopa therapy and that this effect is compensated with higher prescribed doses of amantadine, selegiline, and possibly other anti-Parkinsonian drugs. The shorter survival period associated with the $\mathrm{C}$ allele of rs622342 suggests that multiple anti-Parkinsonian drugs are involved and that the lower response to one or more antiParkinsonian drugs is not compensated by higher prescribed doses of other drugs.

More transporters than OCT1 are involved in the transportation of anti-Parkinsonian drugs, such as OCT2 and OCT3 and the L-type amino acid transporter1 and 2. These transporters differ in their expression throughout the body, distinguishing different roles in absorption, distribution, and elimination of levodopa. OCT1 is expressed in the liver, small intestine, and, in small amounts, in the brain [1]. We do not know the location of transporters that are responsible for the observed difference and why an effect on OCT1 transporter capacity is not compensated for by other transporters. It is possible that the rs622342 $\mathrm{C}$ allele results in a reduced antiParkinson drug uptake from the small intestine resulting in a decreased biological availability [12]. However, OCT1 is also located in small amounts in the brains, and we cannot exclude that these transporters are for example the ratelimiting step for uptake of anti-Parkinson drugs in the brain and responsible for the difference in anti-Parkinson drug response between rs622342 genotypes [1].

The rs622342 polymorphism is located in an intron region, not coding for an amino-acid change. Most likely, this polymorphism is in linkage disequilibrium with a functional polymorphism, although we cannot exclude that it has a direct effect, for example, by affecting gene expression.

In population-based studies, bias may affect the obtained results. We believe that bias in our study is minimal. We identified all incident levodopa users in the Rotterdam Study, and information was collected prospectively, without prior knowledge of the study hypothesis, making selection and information bias unlikely. We did not find any difference in time to levodopa therapy, prior use of other anti-Parkinson medication, or prescribed doses of antiParkinson drugs at start of levodopa therapy, making it unlikely that the rs622342 polymorphism affects the progression to Parkinson's disease. We did not have information on the indication of levodopa use; therefore, we cannot stratify on these indications.

Nine participants were prescribed dopamine agonists and did not receive prescriptions for levodopa during follow-up. These participants were not included in this study, because 
we cannot exclude that the dopamine agonists were prescribed for other indications.

The number of participants in this study was limited; therefore, we cannot exclude that our results were a falsepositive finding, and replication of these results in another cohort is indicated.

To conclude, in this population-based cohort study, the rs622342 minor C variant allele in the SLC22A1 gene, encoding OCT1, was associated with higher prescribed doses of anti-Parkinsonian drugs and shorter survival time after start of levodopa therapy. A possible explanation is that this variant allele is associated with a decreased efficacy of the transportation of anti-Parkinsonian drugs by OCT1 to the brain. The results suggest that patients with the $\mathrm{AC}$ or $\mathrm{CC}$ genotype have less response to these drugs and more severe symptoms, resulting in a shorter survival period.

Acknowledgements This study was supported by the Netherlands Genomics Initiative/Netherlands Organisation for Scientific Research project nr. 050-060-810.

Conflict of Interest The authors declare that they have no conflict of interest.

Open Access This article is distributed under the terms of the Creative Commons Attribution Noncommercial License which permits any noncommercial use, distribution, and reproduction in any medium, provided the original author(s) and source are credited.

\section{References}

1. Koepsell H, Lips K, Volk C (2007) Polyspecific organic cation transporters: structure, function, physiological roles, and biopharmaceutical implications. Pharm Res 24:1227-1251
2. Jonker JW, Schinkel AH (2004) Pharmacological and physiological functions of the polyspecific organic cation transporters: OCT1, 2, and 3 (SLC22A1-3). J Pharmacol Exp Ther 308:2-9

3. Breidert T, Spitzenberger F, Grundemann D, Schomig E (1998) Catecholamine transport by the organic cation transporter type 1 (OCT1). Br J Pharmacol 125:218-224

4. Okura T, Ito R, Ishiguro N, Tamai I, Deguchi Y (2007) Bloodbrain barrier transport of pramipexole, a dopamine D2 agonist. Life Sci 80:1564-1571

5. Ishiguro N, Saito A, Yokoyama K, Morikawa M, Igarashi T, Tamai I (2005) Transport of the dopamine D2 agonist pramipexole by rat organic cation transporters OCT1 and OCT2 in kidney. Drug Metab Dispos 33:495-499

6. Goralski KB, Lou G, Prowse MT, Gorboulev V, Volk C, Koepsell H, Sitar DS (2002) The cation transporters rOCT1 and rOCT2 interact with bicarbonate but play only a minor role for amantadine uptake into rat renal proximal tubules. J Pharmacol Exp Ther 303:959-968

7. Gomes P, Serrao MP, Viera-Coelho MA, Soares-da-Silva P (1997) Opossum kidney cells take up L-DOPA through an organic cation potential-dependent and proton-independent transporter. Cell Biol Int 21:249-255

8. Pinto-do OP, Soares-da-Silva P (1996) Studies on the pharmacology of the inward transport of L-DOPA in rat renal tubules. Br J Pharmacol 118:741-747

9. Becker ML, Visser LE, van Schaik RH, Hofman A, Uitterlinden AG, Stricker BH (2009) Genetic variation in the organic cation transporter 1 is associated with metformin response in patients with diabetes mellitus. Pharmacogenomics J 9:242247

10. Hofman A, Breteler MM, van Duijn CM, Janssen HL, Krestin GP, Kuipers EJ, Stricker BH, Tiemeier H, Uitterlinden AG, Vingerling JR, Witteman JC (2009) The Rotterdam Study: 2010 objectives and design update. Eur J Epidemiol 24:553572

11. International HapMap Consortium (2003) The International HapMap Project. Nature 426:789-796

12. Martel F, Grundemann D, Calhau C, Schomig E (2001) Apical uptake of organic cations by human intestinal Caco-2 cells: putative involvement of ASF transporters. Naunyn Schmiedebergs Arch Pharmacol 363:40-49 